\title{
The association between HIV infection and periprosthetic joint infection following total hip replacement in young adults
}

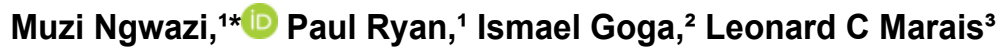 \\ 1 Inkosi Albert Luthuli Hospital, School of Clinical Medicine, Division of Medicine, Department of Orthopaedic Surgery, University of KwaZulu-Natal, Durban, \\ South Africa \\ 2 Inkosi Albert Luthuli Hospital, Durban, South Africa \\ 3 School of Clinical Medicine, Division of Medicine, Department of Orthopaedic Surgery, University of KwaZulu-Natal, Durban, South Africa
}

*Corresponding author: ngmuzi@gmail.com and muzingw@ialch.co.za

Citation: Ngwazi M, Ryan P, Goga I, Marais LC. The association between HIV infection and periprosthetic joint infection following total hip replacement in young adults. SA Orthop J 2021;20(2):76-80. http://dx.doi. org/10.17159/2309-8309/2021/ v20n2a2

Editor: Dr Michael Held, University of Cape Town, South Africa

Received: July 2020

Accepted: November 2020

Published: May 2021

Copyright: (c) 2021 Ngwazi M

This is an open-access article distributed under the terms of the Creative Commons

Attribution Licence, which permits unrestricted use, distribution and reproduction in any medium, provided the original author and source are credited.

Funding: No funding was received for this study.

Conflict of interest: The authors declare they have no conflicts of interest that are directly or indirectly related to the research.

\section{Abstract \\ Background}

The HIV burden in South Africa is high. HIV-positive patients are at risk of developing avascular necrosis of the femoral head, necessitating total hip arthroplasty (THA) at a relatively young age. The primary aim of this study was to investigate the relationship between HIV infection and the risk of periprosthetic joint infection (PJI) in young adults following total hip replacement. Secondly, we aimed to evaluate the association of HIV infection with venous thromboembolic events, reoperation and revision surgery.

\section{Methods}

We undertook a retrospective cohort study involving patients under the age of 55 years who underwent THA between 2009 and 2016 at a tertiary level arthroplasty unit. In total, 290 cases in 213 patients were analysed, with 77 patients requiring bilateral THA. The median age of patients was 43 years (interquartile range [IQR] 39-48, range 26-54 years). Sixty-two per cent of patients were HIV positive $(n=180)$ with a median CD4 count of 520 cells $/ \mathrm{mm}^{3}$ (IQR 423-659, range 238-1308 cells $\left./ \mathrm{mm}^{3}\right)$. Seventy-eight per cent $(n=141)$ of the HIV-positive patients were on antiretroviral medication before surgery. Almost all cases performed in the HIV-positive group were for avascular necrosis $(n=178,99 \%)$.

\section{Results}

At a median follow-up of four years (range 2-10) there were no revisions in either group. The incidence of PJI was $1.1 \%$ in the HIV-positive group vs $0.9 \%$ in the HIV-negative group. The odds ratio for the development of PJI in HIV-positive patients was $1.22(95 \% \mathrm{Cl} 0.11$ to 13.67 , $p=0.869$ ). There was no association between the CD4 count of HIV-positive patients and the development of $\mathrm{PJI}(p=0.171)$. There was no difference in the rate of venous thromboembolic events between the HIV-positive and HIV-negative groups ( $4 \%$ vs $6 \%, p=0.340$ ).

\section{Conclusion}

We report on a cohort of young adult patients who underwent THA. Patients living with HIV infection were not found to be at increased risk for PJI following THA, when compared to HIVnegative patients. The premise that HIV infection increases the risk for PJI following THA remains to be substantiated. This study was underpowered in terms of the primary outcome measure and larger studies are required to verify these findings.

Level of evidence: Level 4

Keywords: total hip arthroplasty, HIV, AIDS, avascular necrosis, hip, periprosthetic joint infection 


\section{Introduction}

The burden of HIV infection in South Africa in general, and in KwaZulu-Natal in particular, is high. Hip disease (avascular necrosis) associated with HIV infection is common, and patients present at a relatively young age requiring total hip arthroplasty (THA).

Although THA is a well-established surgical procedure with excellent outcomes reported in the treatment of advanced hip disease, there is scant information on the outcomes in HIVpositive patients, and controversy remains regarding the risks of complications, specifically periprosthetic joint infection (PJI). ${ }^{1}$ Current literature is contradictory, and studies lack long-term follow-up. Most reports emanate from high-income countries, and these results may not necessarily be applicable to a developing world setting. ${ }^{2,3}$ Many of the previous studies on the topic focused on haemophilic patients and intravenous drug abusers with HIV co-infection, the results of which may not be applicable to other HIV-positive patients. ${ }^{4-8}$

In our unit, we encounter a large number of relatively young patients with HIV requiring total hip replacement. The subset of younger patients requiring THA present their own challenges as they have increased activity levels, physical demands, and need longevity of their implanted components.

The primary aim of this study was to compare the risk of PJI in HIV-positive and HIV-negative young adult patients, following total hip replacement surgery. Secondly, we aimed to evaluate the association of HIV infection with venous thromboembolic events (VTE), reoperation and revision surgery.

\section{Methods}

We performed a retrospective cohort study. Subjects were identified from a prospectively maintained electronic database. All patients below the age of 55 years who received THA at our institution between 2009 and 2016 were deemed eligible for inclusion. Patients with less than a two-year follow-up, incomplete data or an unknown HIV status were excluded. Patient notes were canvassed for demographics, diagnosis, risk factors, HIV status, antiretroviral (ARV) treatment and CD4 count, and extracted. In terms of outcome, we recorded mortality, the development of PJI, deep venous thrombosis or pulmonary embolism, the need for reoperation or revision and the Merle d'Aubigné Hip Score as recorded at six months postoperatively. ${ }^{9}$ We also evaluated radiographs for evidence of radiographic incorporation, as well as complications such as subsidence or loosening. PJI was defined according to the criteria provided by the International Consensus Group. ${ }^{10}$ Superficial infection was defined as a superficial incisional surgical site infection (SSI), as defined by the US Centers for Disease Control and Prevention (CDC) that did not meet the diagnostic criteria for periprosthetic infection. ${ }^{11}$ Deep venous thrombosis and pulmonary embolism were diagnosed on the basis of clinical findings along with confirmatory laboratory and radiological findings. Reoperation was defined as any surgical procedure necessitated by the index THA or any subsequent reoperations, while revision implies a reoperation involving removal and reimplantation of the acetabular cup and/or femoral stem (i.e., isolated acetabular liner exchange was not counted as a revision procedure).

From the review period a total of 316 records were identified and screened. Twenty-six patients were excluded on the basis that their HIV status was not known, leaving 290 cases in 213 patients included for analysis. Each THA procedure was treated as a unique case, with 77 patients requiring bilateral THA. The median age of patients was 43 years (interquartile range [IQR] 39-48, range
26-54 years). Forty-six per cent $(n=132)$ of cases were male, the right side was operated in 165 cases $(57 \%$ ) and staged bilateral hip replacement was required in 36 patients (14\%). Avascular necrosis (AVN) was the indication for surgery in $78 \%$ (225/290) of cases (Figure 1) and osteoarthritis was the indication for surgery in the remainder. In terms of additional risk factors, $21 \%$ of cases were current cigarette smokers, $22 \%$ current alcohol users and $23 \%$ had a history of previous corticosteroid use. Sixty-two per cent of patients were HIV positive (180/290) with a median CD4 count of 520 cells $/ \mathrm{mm}^{3}$ (IQR 423-659, range 238-1 $308 \mathrm{cells} / \mathrm{mm}^{3}$ ). The majority of these patients $78 \%(n=141)$ were on ARV medication prior to the THA. Almost all cases performed in the HIV-positive group were for AVN ( $n=178,99 \%)$.

\section{Operative procedure}

A standard preoperative assessment protocol was used for all patients admitted for joint replacement, irrespective of HIV status. This included a clinical examination, standard AP and lateral X-rays, baseline blood parameters and urine for microscopy, culture and sensitivity. All patients who were planned for joint arthroplasty were encouraged to undergo voluntary counselling and testing (VCT) for HIV, but this was not deemed as mandatory. In HIV-positive patients, a CD4 blood count was obtained. Unit protocol is to

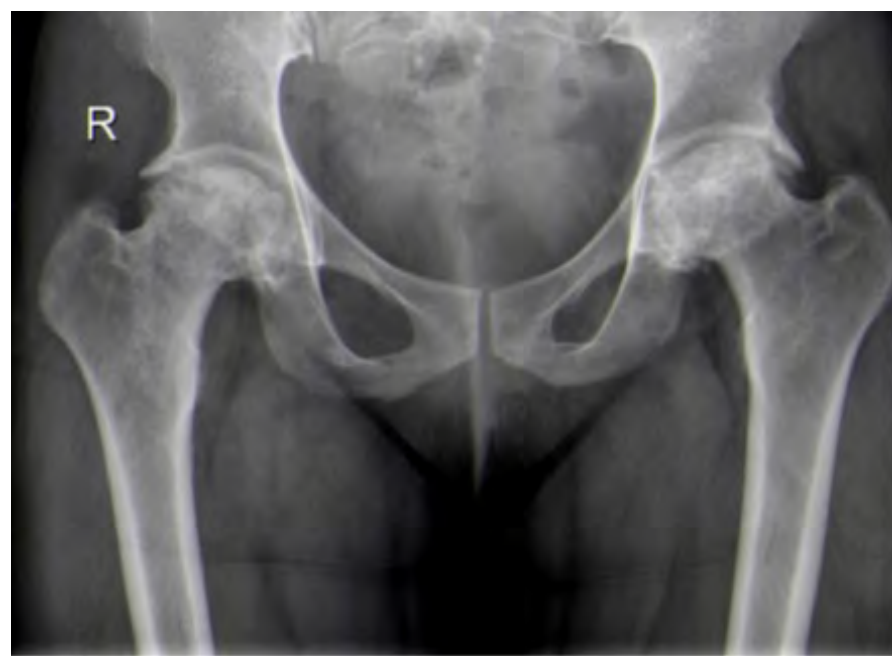

Figure 1. Case example illustrating typical findings in a patient with bilateral AVN

Table I: Comparison of demographic and clinical characteristics of the HIV-positive and HIV-negative groups

\begin{tabular}{|c|c|c|c|}
\hline Characteristic & $\begin{array}{l}\text { HIV-positive } \\
\text { n (\%) }\end{array}$ & $\begin{array}{c}\text { HIV-negative } \\
\text { n (\%) }\end{array}$ & p-value ${ }^{i}$ \\
\hline Cases & $180(62 \%)$ & $110(38 \%)$ & - \\
\hline $\begin{array}{l}\text { Age (median years } \\
\text { [IQR]) }\end{array}$ & $42(38-48)$ & $43(40-48)$ & $0.115^{\mathrm{ii}}$ \\
\hline Male sex & $49(27 \%)$ & $83(75 \%)$ & $<0.001$ \\
\hline Left side operated & $72(40 \%)$ & $53(48 \%)$ & 0.172 \\
\hline Bilateral THA required & $54(30 \%)$ & $23(16 \%)$ & 0.009 \\
\hline \multicolumn{4}{|l|}{ Indication for surgery } \\
\hline Avascular necrosis & $178(99 \%)$ & $56(43 \%)$ & $<0.001$ \\
\hline \multicolumn{4}{|l|}{ Comorbidities } \\
\hline Smoking & $43(24 \%)$ & $18(16 \%)$ & 0.127 \\
\hline Chronic alcohol use & $38(21 \%)$ & $24(22 \%)$ & 0.887 \\
\hline Corticosteroid use & $37(21 \%)$ & $28(25 \%)$ & 0.332 \\
\hline
\end{tabular}

i: Pearson's chi-squared test, unless stated otherwise; ii: Mann-Whitney test 
proceed to joint replacement only if the CD4 count is more than 350 cells $/ \mathrm{mm}^{3}$; however, there were six patients in which the CD4 count was less than 350 cells $/ \mathrm{mm}^{3}$ (but more than $200 \mathrm{cells} / \mathrm{mm}^{3}$ ). Due to the severity of symptoms and the resultant incapacity, it was decided to proceed with surgery in these few patients. Three of these patients required bilateral THA and the lower CD4 count was recorded at the time of the second procedure.

There were no cases performed on patients with BMl>40 kg/m², as this is also a unit protocol exclusion. Patients requiring bilateral THA were staged at separate sittings. The prosthesis used was an uncemented Corail ${ }^{\circledR} /$ Pinnacle System (De Puy, Warsaw) in all patients. The use of drains was at the discretion of the operating surgeon. Three doses of prophylactic intravenous antibiotics were administered in all cases postoperatively. Thromboprophylactic therapy was initiated 12 hours post-surgery in the form of low molecular weight heparin (40 mg enoxaparin daily) which then converted to oral rivaroxaban $10 \mathrm{mg}$ daily within two days and was continued for a total of 14 days post-surgery. Tranexamic acid was not used in this cohort of cases. Patients were discharged on crutches once mobilised and were allowed to weight-bear as tolerated. The patients were followed up clinically and radiologically at six weeks, six months, and annually thereafter.

\section{Statistical methods}

Statistical analysis was performed using Stata 15.0 (StataCorp. College Station, Texas). Continuous variables were reported as mean ( \pm standard deviation [SD]) if normally distributed or median (with IQR) if skewed and categorical variables as number and percentages, unless otherwise stated. Apart from the Merle d'Aubigné Hip Score, all continuous variables were non-normally distributed. Differences in continuous variables were compared with the use of the Student's t-test or Mann-Whitney test, as appropriate. Categorical data were compared using the Fisher's exact test or the chi-squared test. The association between PJI and HIV status was tested using binominal logistic regression analysis. All tests were two-sided, and the level of significance was set at $p<0.05$.

\section{Results}

The median follow-up was four years (IQR 2-5, range 2-10 years). The follow-up was marginally longer in the HIV-positive group $(p=0.021)$. Comparative characteristics of the HIV-positive and HIV-negative groups is provided in Table I. The HIV-positive group also contained fewer male patients $(27 \%$ vs $75 \%$, $p<0.001)$, more cases with $\mathrm{AVN}(99 \%$ vs $43 \%, \mathrm{p}<0.001)$ and more cases requiring staged bilateral THA ( $30 \%$ vs $16 \%, p=0.009)$.

With regard to postoperative complications, there were six cases of superficial wound infection (one HIV-positive patient and five HIV-negative patients) which settled with dressings and short courses of antibiotics. There were three cases of periprosthetic joint infection, two of which occurred in HIV-positive patients $(1.1 \%$ in HIV-positive group vs $0.9 \%$ in HIV-negative group, Table II). The odds ratio for the development of PJI in HIV-positive patients was $1.22(95 \% \mathrm{Cl} 0.11$ to $13.67, \mathrm{p}=0.869)$ (Table III). There was no association between the CD4 count of HIV-positive patients and the development of PJI $(p=0.171)$. In addition, patients who were not on ARV medication were not more prone to PJI. All cases of PJI were successfully treated by reoperation in the way of debridement, irrigation, polyethylene exchange and implant retention.

One HIV-positive patient demised seven days post-surgery due to a pulmonary embolism, which was confirmed at post-mortem. Notably, there was no difference in the rate of VTE between the HIV-positive and HIV-negative groups ( $4 \%$ vs $6 \%, p=0.340)$.
Table II: Comparison of outcome measures and follow-up in the two groups

\begin{tabular}{|l|c|c|c|}
\hline \multicolumn{1}{|c|}{ Characteristic } & $\begin{array}{c}\text { HIV-positive } \\
\mathbf{n}(\%)\end{array}$ & $\begin{array}{c}\text { HIV-negative } \\
\mathbf{n}(\%)\end{array}$ & p-value \\
\hline $\begin{array}{l}\text { Cases } \\
\text { Complications }\end{array}$ & $180(62 \%)$ & $110(38 \%)$ & - \\
\hline $\begin{array}{l}\text { Periprosthetic joint } \\
\text { infection }\end{array}$ & $2(1 \%)$ & $1(1 \%)$ & $0.869^{\text {iii }}$ \\
\hline $\begin{array}{l}\text { Aseptic loosening } \\
\text { Venous } \\
\text { thromboembolism }\end{array}$ & $0(0 \%)$ & $0(0 \%)$ & - \\
\hline \begin{tabular}{l} 
Reoperation \\
\hline Mortality
\end{tabular} & $2(1 \%)$ & $7(6 \%)$ & 0.345 \\
\hline $\begin{array}{l}\text { Follow-up duration } \\
\text { (median years [IQR]) }\end{array}$ & $1(1 \%)$ & $0(0 \%)$ & 1.000 \\
\hline PROMiv (median [IQR]) & $14(13-15)$ & $14(13-15)$ & 0.154 ii \\
\hline
\end{tabular}

i: Pearson's chi-squared test, unless stated otherwise; ii: Mann-Whitney test; iii: Binominal logistic regression; iv: Merle d'Aubigné Hip Score

Table III: Odds ratios for the development of complications in HIV-positive patients compared with HIV-negative patients

\begin{tabular}{|l|c|c|c|}
\hline \multicolumn{1}{|c|}{ Complication } & $\begin{array}{c}\text { Odds } \\
\text { ratio }\end{array}$ & $\begin{array}{c}95 \% \\
\text { confidence } \\
\text { interval }\end{array}$ & p-value \\
\hline Periprosthetic joint infection & 1.22 & $0.11-13.67$ & 0.869 \\
\hline Venous thromboembolism & 0.59 & $0.20-1.7$ & 0.345 \\
\hline Reoperation & 1.22 & $0.11-13.67$ & 0.869 \\
\hline Aseptic loosening & 1 & - & - \\
\hline Mortality & 1 & - & - \\
\hline
\end{tabular}

Radiological review confirmed stem integration on all X-rays at last follow-up with no signs of loosening or subsidence. There were no dislocations during the follow-up period. The patient-reported outcome, as represented by the Merle d'Aubigné Hip Score at six months postoperatively, was similar among HIV-positive and HIVnegative patients $(p=0.154)$.

\section{Discussion}

South Africa has a high burden of HIV disease. Prevalence of up to $46 \%$ in patients between the ages of 25 and 40 years has been reported..$^{12}$ Antiretroviral therapy (ART) has made a drastic impact on adult life expectancy, with a reported increase of 17 years from 2001 to $2014 .{ }^{13}$ However, while there have been significant improvements in survival, there remains a considerable burden of morbidity in patients living with HIV, including osteoarticular manifestations.

AVN of the hip is up to 40 times more likely to develop in HIVinfected individuals compared to the general population. ${ }^{14}$ The disease may progress faster and have more intense symptoms in people living with HIV. It was previously thought that ART might be associated with this increased risk; however, recent studies have called this long-held belief into question. ${ }^{15} \mathrm{~A}$ meta-analysis performed in 2014 reported a two-fold increase in the odds of AVN in patients exposed to protease inhibitors. ${ }^{16}$ Conversely, a large international cohort study found that no ARVs were associated with osteonecrosis and that the increased risk may be related to HIV infection itself. ${ }^{17}$

The current literature on the outcomes and complications following THA in patients with HIV infection is conflicting. A nationwide survey from the USA involving 9275 patients with HIV who 
underwent THA before 2010 showed an increased risk of both major and minor complication in HIV patients. ${ }^{18}$ Early reports on arthroplasty in HIV-positive patients also raised concerns about the possible increased risk of complications, early failure and periprosthetic infection. ${ }^{19,20}$

The combination of these factors, namely the high prevalence of HIV infection, an increased need for THA and the concerns about an increased risk of PJI, along with the dearth of data from the developing world makes this study particularly relevant in the South African clinical setting. We found no difference in the rate of PJI, VTE, aseptic loosening, patient-reported outcome at six months, reoperation, revision or mortality between the two groups at a median four-year follow-up. Notably, $78 \%$ of HIV-positive patients were on ARV medication at the time of surgery and only patients with an absolute CD4 count above $200 \mathrm{cells} / \mathrm{mm}^{3}$ were considered eligible for surgery. In addition, we found no association between HIV-positive patients' CD4 count or ARV status and the development of infection.

The existing data on the infection risk in HIV-positive patients undergoing arthroplasty is conflicting. A recent meta-analysis on the outcome of knee and hip arthroplasty in HIV patients found an elevated risk of complications in HIV-positive patients (relative risk = 2.28; 95\% Cl 2.14-2.43). ${ }^{21}$ However, the authors were not able to analyse the infection risk separately due to heterogenous reporting of data on the topic. Some of the early studies on arthroplasty in HIV patients did have a control group., ${ }^{2,19}$ Others included patients with additional risk factors for infection like haemophilia or intravenous drug abuse (IVDA).4-8 Naziri and colleagues interrogated a large US database and found an increased risk of 'wound infection' $(0.7 \%$ vs $0.2 \%$; OR 2.38 ; $95 \%$ $\mathrm{Cl}$ 1.32-4.30). ${ }^{18}$ While the authors defined 'implant infection' as a minor complication, these were not reported in the results of the study. The authors also recognised that the limitations of their study included the fact that infections could not adequately be assessed using the inpatient data alone and that the data was restricted to the diagnosis recorded during the hospital stay without providing further data relating to the long-term outcomes. While this study, therefore, provides compelling evidence of an increased risk of complications in HIV-infected patients, it is difficult to draw conclusions on the association with infection specifically. In 2013 Capogna et al. suggested that the rate of early joint infection might be lower than previously suggested. ${ }^{22}$

Our finding that HIV infection may not necessarily be associated with an increased risk for infection is certainly not a novel one. Graham et al. reported the outcome of 43 THAs in HIV patients without haemophilia or IVDA and found no early or late infections at a mean 3.5-year follow-up. ${ }^{3}$ They also had no revision cases and no signs of aseptic loosening. While a study by Brijlall also showed good outcomes, patients were given antibiotics for an extended period. ${ }^{2}$ Similarly, Graham et al. also routinely prescribed long-term antibiotic cover in the form of co-cotrimoxazole. ${ }^{3}$ We used prophylactic antibiotics for a maximum of 24 hours only. This appears not to have been associated with an increased infection rate, but further studies would be required to confirm this.

There are numerous other studies that have also failed to show an increased risk of infection. Tornero et al. found no significant difference in terms of either outcome or complications when comparing 18 cases of THA in HIV-positive patients with those of 27 HIV-negative cases. ${ }^{23}$ Snir et al. reported one deep infection out of 41 non-haemophilic HIV-positive cases. Their study unfortunately did not have a control group. ${ }^{24}$ Powell and colleagues analysed their results over a 27 -year period in haemophiliacs with HIV and noted three infections per 205 joint-years in the HIV-positive group with no increased risk for PJI. ${ }^{5}$ Falakassa et al. reported that, at a mean follow-up of 14 months, they encountered no PJls in 31 total hip arthroplasties in non-haemophiliac patients who were all on highly active anti-retroviral therapy (HAART). ${ }^{25}$ Wang and coworkers also reported in no infection in ten hip, knee and shoulder arthroplasties at an average follow-up of 38 months. ${ }^{26}$ Similarly, Lubega and colleagues from Malawi reported no deep sepsis among 14 HIV patients who underwent arthroplasty surgery. ${ }^{27}$ Perhaps most striking is the systematic review by Enayatollahi and colleagues which concluded that patients on HAART and optimised comorbidities appear to have a lower rate of PJI. ${ }^{28}$ These results are not dissimilar from the findings of our study.

In other orthopaedic sub-disciplines, the assumption that HIV infection increases the risk of infection has also been questioned. A recent meta-analysis looking at the risk of infection following fracture surgery noted that in the era of antiretroviral therapy, HIV-infected patients did not appear to have an elevated risk of fracture-related infection in open or closed fractures. ${ }^{29}$

Aside from the risk of infection, there are concerns about several other complications related to arthroplasty in HIV-positive patients. There is data suggesting a greater tendency to thromboembolic phenomena in HIV-positive patients (two- to ten-fold increased risk), with patients younger than 50 years appearing to be particularly succeptible. ${ }^{30}$ However there are few reports from the developing world regarding this. In our study period, one patient demised seven days postoperatively. She had a history of treated pulmonary tuberculosis for more than ten years previously. The preoperative CD4 count was 944 cells $/ \mathrm{mm}^{3}$ and BMI was 26. She was started on thromboprophylaxis and mobilised as per protocol from day 1. Post-mortem results confirmed a pulmonary embolism. The current study did not show any increased incidence in VTE using a standardised prevention strategy.

Concerns have also been raised regarding the possibility of stem subsidence or periprosthetic fractures related to the decreased bone quality associated with HIV infection. ${ }^{15,31,32}$ While some authors have opted for cemented prostheses using antibioticimpregnated bone cement, we opted for uncemented prostheses in all cases. ${ }^{3}$ A review of the postoperative X-rays of the hip and pelvis in these patients showed excellent osteointegration (Figure 2), with no signs of loosening or stem subsidence. Radiological assessment showed no differences between HIV and non-HIV patients. The details of these radiological features have been published previously. ${ }^{33}$ We believe that both uncemented and cemented stems can be used with good outcomes, although the limitation of our relatively short follow-up needs to be kept in mind. The choice of implants should follow the standard selection criteria based mainly on the quality of bone of that patient.

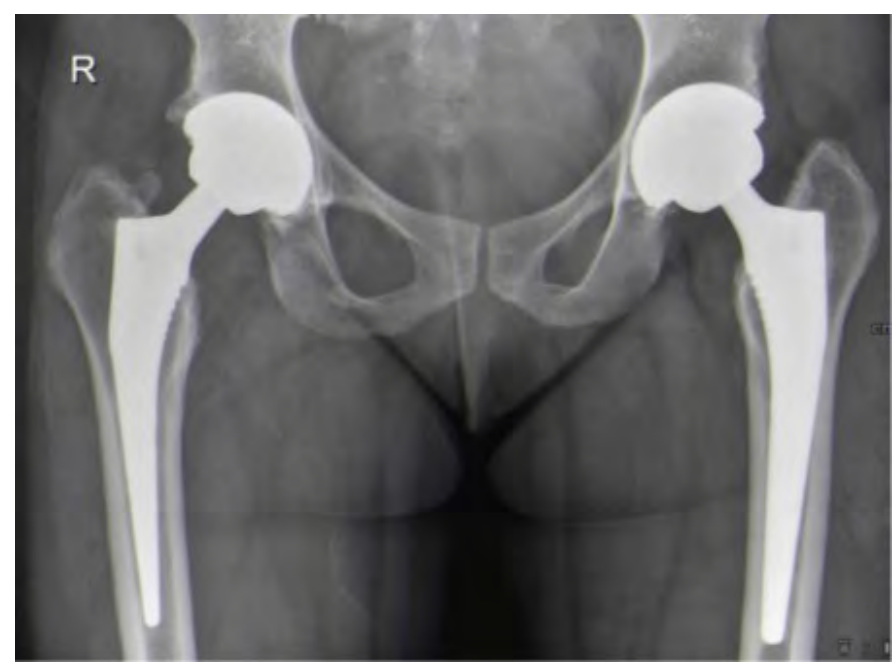

Figure 2. Case example illustrating bilateral total hip arthroplasties with no signs of loosening at four-year review 
There were several limitations to this study, primarily due to its retrospective nature. The preoperative hip functional outcomes scores were not available in some of the patients and we could only report consistently on the six months postoperative measure. Therefore, we are not able to make any conclusions in terms of the functional outcome in HIV-positive versus HIV-negative patients. Furthermore, there were insufficient data relating to risk factors such as obesity, diabetes mellitus and nutritional status to allow sub-group analysis. While this may be the largest cohort study of its nature, it remains underpowered for the primary outcome measure. With a low event rate a much larger sample size will be needed to provide high level evidence. The relatively short median followup of four years needs to be taken into account when considering aseptic loosening and the risk of periprosthetic fractures.

\section{Conclusion}

We report on a cohort of young adult patients who underwent THA. Patients living with HIV infection were not found to be at increased risk for PJI following THA, when compared to HIV-negative patients. The premise that HIV infection increases the risk for PJI following THA remains to be substantiated. This study was underpowered in terms of the primary outcome measure and larger studies are required to verify these findings.

\section{Ethics statement}

The author/s declare that this submission is in accordance with the principles laid down by the Responsible Research Publication Position Statements as developed at the 2nd World Conference on Research Integrity in Singapore, 2010.

Prior to commencement of the study ethical approval was obtained from the following ethical review board: UKZN BREC, reference number BE079/16.

All procedures were in accordance with the ethical standards of the responsible committee on human experimentation (institutional and national) and with the Helsink Declaration of 1975 , as revised in 2008 .

\section{Declaration}

The authors declare authorship of this article and that they have followed sound scientific research practice. This research is original and does not transgress plagiarism policies

\section{Author contributions}

MN: Study conceptualisation, data capture, data analysis, first draft preparation, manuscript revision

PR: First draft preparation, language and grammar correction, data analysis IG: First draft preparation, language and grammar correction, data analysis LCM: Data analysis and final draft preparation

\section{ORCID}

Ngwazi M 19 https://orcid.org/0000-0003-3831-3830

Ryan P https://orcid.org/0000-0002-0957-6482

Marais LC 19 https://orcid.org/0000-0002-1120-8419

\section{References}

1. Pietrzak JRT, Maharaj Z, Mokete L, Sikhauli N. Human immunodeficiency virus in total hip arthroplasty. EFORT Open Rev. 2020;5:161-71.

2. Brijlall S. Hip arthroplasty in HIV-infected patients. SA Orthop J. 2008;7(1):10-16

3. Graham SM, Lubega N, Mkandawire N, Harrison WJ. Total hip replacement in HIV positive patients. J Bone Joint Surg (Br). 2014;96-B(4):462-66.

4. Hicks JL, Ribbans WJ, Buzzard B, et al. Infected joint replacements in HIV positive patients with haemophilia. J Bone Joint Surg (Br) 2001;83-B(7):1050-54.

5. Powell DL, Whitener CJ, Dye CE, et al. Knee and hip arthroplasty infection rates in persons with haemophilia: a 27-year single-centre experience during the HIV epidemic. Haemophilia. 2005;11(3):233-39.

6. Goddard NJ, Mann HA, Lee CA. Total knee replacement in patients with end-stage haemophilic arthropathy: 25-year results. J Bone Joint Surg (Br). 2010;92-B(8):1085-89.

7. Habermann B, Eberhardt $C$, Kurth AA. Total joint replacement in HIV positive patients. J Infection. 2008;57(1):41-46.
8. Ragni MV, Crossett LS, Herndon JH. Post-operative infection following orthopaedic surgery in human immunodeficiency virus-infected haemophiliacs with CD4 counts < or = 200/mm3. J Arthroplasty. 1995;10(6):716-21.

9. Ugino F, Carolina R, Alves D. Evaluation of the reliability of the modified Merle d'Aubigne and pastel method. Acta Ortop Bras. 2012:20(4):213-17.

10. Parvizi J, Gehrke T. Definition of periprosthetic joint infection. J Arthroplasty. 2014;29:1331.

11. Dindo D, Demartines N, Clavien PA. Classification of surgical complications: a new proposal with evaluation in a cohort of 6336 patients and results of a survey. Ann Surg. 2004;240:205-13.

12. De Oliveira $T$, Kharsany ABM, Gräf T, et al. Transmission networks and risk of HIV infection in KwaZulu-Natal, South Africa: a community-wide phylogenetic study. Lancet HIV. 2017;4:e41-50.

13. Zaidi J, Grapsa E, Tanser F, Newell ML, Barnighausen T. Dramatic increase in HIV prevalence after scale-up of antiretroviral treatment. AIDS. 2013;27:2301-305.

14. Mazzotta E, Agostinone A, Rosso R, et al. Osteonecrosis in human immunodeficiency virus (HIV)-infected patients: a multicentric case-control study. J Bone Miner Metab. 2011;29(3):383-88.

15. Mary-Krause M, Billaud E, Poizot-Martin I, et al. Risk factors for osteonecrosis in HIV-infected patients: impact of treatment with combination antiretroviral therapy. AIDS. 2006;20(12):1627-35.

16. Permpalung N, Ungprasert P, Summachiwakij S, Leeaphorn N, Knight EL. Protease inhibitors and avascular necrosis: A systematic review and meta-analysis. Int J Antimicr Agents. 2014;44:93-95.

17. Borges $\mathrm{AH}$, Hoy J, Florence $\mathrm{E}$, et al. Anti-retrovirals, fractures, and osteonecrosis in a large international HIV cohort. Clin Inf Dis. 2017;64(10):1413-21.

18. Naziri Q, Boylan MR, Issa KM, et al. Does HIV infection increase the risk of perioperative complications after THA? A nationwide database study. Clin Orthop Rel Res. 2015;473(2):581-86.

19. Parvizi J, Sullivan TA, Pagnano MW, Trousdale RT, Bolander ME. Total joint arthroplasty in human immunodeficiency virus-positive patients: An alarming rate of early failure. J Arthroplasty. 2003;18(3):259-64.

20. Lehman CR, Ries MD, Paiement GD, Davidson AB. Infection after total joint arthroplasty in patient with human immunodeficiency virus or intravenous drug use. J Arthroplasty. 2001;16(3):330-35.

21. Dimitriou D, Ramokgopa M, Pietrzak JRT, Van der Jagt D, Mokete P. Human immunodefiniency virus infection and hip and knee arthroplasty. JBJS Reviews. 2017;5(9):e8.

22. Capogna BM, Lovy A, Blum $Y$, et al. Infection rate following total joint arthroplasty in HIV population. J Arthroplasty. 2013;25(8):1254-58.

23. Tornero E, García S, Larouse M, et al. Total hip arthroplasty in HIV-infected patients: a retrospective, controlled study. HIV Med. 2012;13(10):623-29.

24. Snir N, Wolfson TS, Schwarzkopf R, et al. Outcomes of total hip arthroplasty in human immunodeficiency virus-positive patients. J Arthroplasty. 2014;29:157-61.

25. Falakassa J, Diaz A, Schneiderbauer M. Outcomes of total joint arthroplasty in HIV patients. lowa Orthop J. 2014;34:102-106

26. Wang TI, Chen CF, Chen WM, et al. Joint replacement in human immunodeficiency virus-infected patients. J Chin Med Assoc 2012;75(11):595-99.

27. Lubega N, Mkandawire NC, Sibande GC, Norrish AR, Harrison WJ. Joint replacement in Malawi. J Bone Joint Surg Br. 2009;91:341-43.

28. Enayatollahi M, Murphy D, Maltenfort M, Parvizi J. Human immunodeficiency virus and total joint arthroplasty: the risk for infection is reduced. J Arthroplasty. 2016:31(10):2146-51.

29. Nieuwoudt L, Rodseth RN, Marais LC. Fracture-related infections in HIV infected patients: A systematic review and meta-analysis. J Orthop. 2020;18:248-54

30. Kiser KL, Badowski ME. Risk factors for venous thrombosis in patients with human immunodeficiency virus infection. Pharmacotherapy. 2010;30(12):1292-302.

31. Martin A, Moore C, Mallon PW, et al. Bone mineral density in HIV participants randomised to raltegravir and lopinavir/ritonavir compared with standard second-line therapy. AIDS. 2013;27(15):2403-11.

32. Sharma A, Tian F, Yin MT, et al. Association of regional body composition with bone mineral density in HIV-infected and HIV-uninfected women: women's interagency HIV study. J Acquir Immune Defic Syndr. 2012;61(4):469-76.

33. Bakkai A, Ryan P, Goga I, et al. Tapered uncemented HA-coated femoral stems: a radiological study. S Afr Orthop J. 2017;16(3)27-30. 